\title{
Oscillation of Half-Linear Differential Equations with Delay
}

\author{
Simona Fišnarová and Robert Mařík \\ Department of Mathematics, Mendel University in Brno, Zemědělská 1, 61300 Brno, Czech Republic
}

Correspondence should be addressed to Robert Mař́ik; marik@mendelu.cz

Received 10 July 2013; Accepted 30 October 2013

Academic Editor: Miroslava Růžičková

Copyright (C) 2013 S. Fišnarová and R. Mařík. This is an open access article distributed under the Creative Commons Attribution License, which permits unrestricted use, distribution, and reproduction in any medium, provided the original work is properly cited.

We study the half-linear delay differential equation $\left(r(t) \Phi\left(x^{\prime}(t)\right)\right)^{\prime}+c(t) \Phi(x(\tau(t)))=0, \Phi(x):=|x|^{p-2} x$, $p>1$. We establish a new a priori bound for the nonoscillatory solution of this equation and utilize this bound to derive new oscillation criteria for this equation in terms of oscillation criteria for an ordinary half-linear differential equation. The presented results extend and improve previous results of other authors. An extension to neutral equations is also provided.

\section{Introduction}

In this paper we study oscillatory properties of the delay second-order half-linear differential equation

$$
\begin{aligned}
& \left(r(t) \Phi\left(x^{\prime}(t)\right)\right)^{\prime}+c(t) \Phi(x(\tau(t)))=0, \\
& \Phi(x):=|x|^{p-2} x, \quad p>1 .
\end{aligned}
$$

We suppose that $r, c, \tau$ are continuous functions defined on $\left[t_{0}, \infty\right)$ such that $r(t)>0, c(t)>0$ for large $t, \tau(t) \leq t$ for all $t$, and $\lim _{t \rightarrow \infty} \tau(t)=\infty$. By $q$ we denote the conjugate number to the number $p$, that is, $q=p /(p-1)$.

Under the solution of (1) we understand any differentiable function $x(t)$ which does not identically equal zero eventually, such that $r(t) \Phi\left(x^{\prime}(t)\right)$ is differentiable and (1) holds for large $t$.

The solution of (1) is said to be oscillatory if it has infinitely many zeros tending to infinity. Equation (1) is said to be oscillatory if all its solutions are oscillatory. In the opposite case, that is, if there exists an eventually positive solution of (1), (1) is said to be nonoscillatory.

It is well known that the behavior of delay differential equations is very different from the behavior of ordinary differential equations. Among others, the Sturm theory fails and oscillatory solutions may coexist with nonoscillatory solutions.

In certain special cases, it is possible to compare asymptotics of (1) with some other simpler equation. One of the typical objects for this comparison is the first order delay differential equation; see, for example, [1-3] for results on comparing (1) or its extension in the form of neutral differential equation with the first order delay differential inequality. Another simpler object than (1) suitable for comparison with (1) is the half-linear second-order ordinary differential equation

$$
\left(r(t) \Phi\left(x^{\prime}(t)\right)\right)^{\prime}+c(t) \Phi(x(t))=0
$$

see, for example, [4-7]. Note that some of these papers deal with a slightly more general equation

$$
\left(r(t) \Phi\left(x^{\prime}(t)\right)\right)^{\prime}+f(t, x(t), x(\tau(t)))=0 .
$$

However, if this more general equation is considered, conditions imposed on the nonlinearity $f$ usually state that (3) is a kind of majorant of (1) (in the sense used in the Sturmian theory of ordinary differential equations) and allow to extend the results readily from (1) to (3). An example of such conditions is

$$
\frac{f(t, u, v)}{\Phi(v)} \geq c(t) \quad \text { or } \quad \frac{f(t, u, v)}{\Phi(u)} \geq c(t)
$$

for some (positive) function $c(t)$ and all positive numbers $u, v$. Note also that some of the above cited papers deal more generally with neutral differential equations and (or) dynamic equations on time scales. 
In this paper we compare (1) with the ordinary halflinear equation of the form (2). To make our paper more readable we restrict our attention to differential equations rather than equations on time scales. An extension of our results to neutral differential equations is provided at the end of this paper.

Let us recall the Riccati technique, which is one of the methods frequently used in oscillation theory of both (1) and (2) (it is easy to see that if $\tau(t)=t$, then (1) reduces to (2)). Suppose that (1) is nonoscillatory and let $x$ be its eventually positive solution. Then the function $w(t)=$ $r(t) \Phi\left(x^{\prime}(t)\right) / \Phi(x(t))$ satisfies the Riccati type equation

$$
w^{\prime}(t)+c(t) \Phi\left(\frac{x(\tau(t))}{x(t)}\right)+(p-1) r^{1-q}(t)|w(t)|^{q}=0 .
$$

The following lemma plays a crucial role in the qualitative theory of half-linear second order ordinary differential equations.

Lemma 1 (see [8, Theorem 2.2.1]). Denote $\mathscr{L}[x]=$ $\left(r(t) \Phi\left(x^{\prime}(t)\right)\right)^{\prime}+c(t) \Phi(x(t))$ and $\mathscr{R}[w]=w^{\prime}+c(t)+(p-$ 1) $r^{1-q}(t)|w|^{q}$. The following statements are equivalent:

(i) (2) is nonoscillatory,

(ii) there is $a \in \mathbb{R}$ and a continuously differentiable function $w:[a, \infty) \rightarrow \mathbb{R}$ such that

$$
R[w](t)=0 \text { for } t \in[a, \infty),
$$

(iii) there is $a \in \mathbb{R}$ and a continuously differentiable function $w:[a, \infty) \rightarrow \mathbb{R}$ such that

$$
R[w](t) \leq 0 \text { for } t \in[a, \infty),
$$

(iv) there is $a \in \mathbb{R}$ and a positive function $x:[a, \infty) \rightarrow \mathbb{R}$ with $r \Phi\left(x^{\prime}\right)$ continuously differentiable such that

$$
\mathscr{L}[x](t) \leq 0 \quad \text { for } t \in[a, \infty) .
$$

As we show below, the assumptions used in the paper ensure that the positive solutions are eventually increasing and concave down. The main step when we compare the ordinary half-linear differential equation and its delay counterpart (1) is to reduce (5) to the Riccati inequality of the form (7). The usual approach on how to remove the term $\Phi(x(\tau(t)) / x(t))$ from (5) is the following lemma, originally proved in [9] and then used in many subsequent papers.

Lemma 2. Suppose that $x$ is a function defined for some $T>0$ such that $x(t) \in C^{2}[T, \infty), x(t)>0, x^{\prime}(t)>0$, and $x^{\prime \prime}(t) \leq 0$ for $t \geq T$. Then, for each $k \in(0,1)$ there exists $T_{k} \geq T$ such that

$$
\frac{x(\tau(t))}{x(t)} \geq k \frac{\tau(t)}{t} \quad \text { for } t \geq T_{k} .
$$

Note that the proof of Lemma 2 does not exploit the fact that $x$ is a solution of (1) and the lemma holds for any positive increasing concave down function. The proof of (9) can be based on the fact that if $x^{\prime \prime}(t) \leq 0$ on $[T, \infty)$ and $x(T) \geq 0$, then $x(t) /(t-T)$ is decreasing with respect to $t$ on $[T, \infty)$ (see [10, Theorem 128]). Thus

$$
\frac{x(\tau(t))}{x(t)} \geq \frac{\tau(t)-T}{t-T}=\frac{\tau(t)}{t} \frac{1-(T / \tau(t))}{1-(T / t)},
$$

where $T \leq \tau(t) \leq t$. Removing the dependence on $T$ may be implemented by using of a constant $k \in(0,1)$. The presence of one of the constants $T$ or $k$ in the estimates (9) and (10) is an important attribute of these estimates. As a consequence, the resulting integral oscillation citeria have to be formulated either with the constant $k \in(0,1)$, or as interval-type or Kamenev-type criteria, where the dependence on $T$ is usually not disturbing. A typical result looks like the following Theorem A.

Theorem A (see [11, Theorem 2.6]). Equation (1) with $r \equiv 1$ is oscillatory if the differential equation

$$
\left(\Phi\left(x^{\prime}(t)\right)\right)^{\prime}+\lambda c(t)\left(\frac{\tau(t)}{t}\right)^{p-1} \Phi(x(t))=0
$$

is oscillatory for some $\lambda \in(0,1)$.

As another particular example of a criterion which suffers from the presence of the constants $m_{i} \in(0,1)$ see $[12$, Theorem 2.1].

The above mentioned disadvantage has been removed for the linear delay equation

$$
x^{\prime \prime}(t)+c(t) x(\tau(t))=0
$$

under the condition

$$
\int_{0}^{\infty} s c(s) d s=\infty .
$$

Opluštil and Šremr utilized in recent papers [13, 14] (12) to derive a sharper estimate than the estimate from Lemma 2. Note that imposing (13) on $c$ does not yield any restriction in oscillation criteria for (12) since (12) is already known to be nonoscillatory if (13) fails. The same approach has been used for linear dynamic equations on time scales by Erbe, Peterson and Saker in [15].

The aim of this paper is to derive a result analogical to the estimate from $[13,14]$ and make it available also for delay halflinear differential equation. The nonlinearity of the equation causes, that the method from $[13,14]$ does not extend to (1) directly and we have to use an indirect approach which originates in the fact that the half-linear extension does not yield (13) as its special case, but includes the term $\tau(s)$ instead of $s$. This estimate suggests a new tool which can be used to improve some oscillation criteria for (1).

\section{Preliminaries}

The proof of the following statement can be found in [16]. 
Lemma 3. Let $x$ be an eventually positive solution of (1). If $\int^{\infty} r^{1-q}(t) d t=\infty$, then $x^{\prime}(t)>0$ for large $t$. Moreover, if $r^{\prime}(t) \geq 0$, then $x^{\prime \prime}(t) \leq 0$ for large $t$.

The following lemma shows that under certain additional conditions we can utilize (1) to derive a sharper version of the estimate from Lemma 2.

Lemma 4. Suppose that (1) is nonoscillatory, and let $x(t)>0$ be a solution of (1). If the conditions

$$
\begin{gathered}
\int^{\infty} r^{1-q}(t) d t=\infty, \quad r^{\prime}(t) \geq 0 \text { for large } t \\
\int^{\infty} c(t) \tau^{p-1}(t) d t=\infty
\end{gathered}
$$

hold, then there exists $T \in \mathbb{R}$ such that

$$
\frac{x(\tau(t))}{x(t)} \geq \frac{\tau(t)}{t}, \quad t \geq T
$$

Proof. Conditions (14) and Lemma 3 imply that there exists $T_{0}$ such that $x(t)>0, x^{\prime}(t)>0, x^{\prime \prime}(t) \leq 0$ for $t \geq T_{0}$.

We show that

$$
t x^{\prime}(t)-x(t) \leq 0
$$

for large $t$. Since $\left(t x^{\prime}(t)-x(t)\right)^{\prime}=t x^{\prime \prime}(t) \leq 0$, it is sufficient to show that (17) holds for some $T_{1} \geq T_{0}$. Suppose, by contradiction, that $t x^{\prime}(t)-x(t)>0$ for all $t \geq T_{0}$. Solving this inequality we get $x(t)>K t$ for $t \geq T_{0}$, where $K=x\left(T_{0}\right) / T_{0}>$ 0 . Hence, there exists $T_{2} \geq T_{0}$ such that

$$
\Phi(x(\tau(t))) \geq K^{p-1}(\tau(t))^{p-1}, \quad t \geq T_{2} .
$$

Since $x$ is a solution of (1), we have

$$
\begin{aligned}
\left(r(t) \Phi\left(x^{\prime}(t)\right)\right)^{\prime} & =-c(t) \Phi(x(\tau(t))) \\
\leq-K^{p-1} c(t)(\tau(t))^{p-1} & \\
t & \geq T_{2} .
\end{aligned}
$$

Integrating the last inequality from $T_{2}$ to $t$ we obtain

$$
\begin{gathered}
r(t) \Phi\left(x^{\prime}(t)\right)-r\left(T_{2}\right) \Phi\left(x^{\prime}\left(T_{2}\right)\right) \\
\leq-K^{p-1} \int_{T_{2}}^{t} c(s)(\tau(s))^{p-1} d s,
\end{gathered}
$$

and from the fact that $r(t) \Phi\left(x^{\prime}(t)\right)$ is positive we get the following finite upper bound for the integral of $c(s)(\tau(s))^{p-1}$ :

$$
\begin{aligned}
& K^{p-1} \int_{T_{2}}^{t} c(s)(\tau(s))^{p-1} d s \\
& \quad \leq r\left(T_{2}\right) \Phi\left(x^{\prime}\left(T_{2}\right)\right)-r(t) \Phi\left(x^{\prime}(t)\right)<r\left(T_{2}\right) \Phi\left(x^{\prime}\left(T_{2}\right)\right)
\end{aligned}
$$

for $t \geq T_{2}$. However the condition (15) ensures that the left hand side of this inequality is unbounded. This contradiction proves (17) for large $t$.

Hence there exists $T_{1} \geq T_{0}$ such that (17) holds for $t \geq T_{1}$. This inequality together with the computation

$$
\left(\frac{x(t)}{t}\right)^{\prime}=\frac{t x^{\prime}(t)-x(t)}{t^{2}} \leq 0, \quad t \geq T_{1},
$$

shows that the function $x(t) / t$ is decreasing on $\left(T_{1}, \infty\right)$. This fact and the fact that $\tau(t) \leq t$ reveal that there exists $T \geq T_{1}$ such that

$$
\frac{x(t)}{t} \leq \frac{x(\tau(t))}{\tau(t)}, \quad t \geq T
$$

which is equivalent to (16).

\section{Oscillation of Delay Differential Equation}

Theorem 5. Suppose that conditions (14) and (15) hold. If the ordinary differential equation

$$
\left(r(t) \Phi\left(x^{\prime}(t)\right)\right)^{\prime}+c(t)\left(\frac{\tau(t)}{t}\right)^{p-1} \Phi(x(t))=0
$$

is oscillatory, then (1) is also oscillatory.

Proof. Suppose, by contradiction, that (1) is nonoscillatory and (24) is oscillatory. Let $x$ be an eventually positive solution of (1). Using Lemma 4 we see that $x$ satisfies the inequality

$$
\left(r(t) \Phi\left(x^{\prime}(t)\right)\right)^{\prime}+c(t)\left(\frac{\tau(t)}{t}\right)^{p-1} \Phi(x(t)) \leq 0
$$

and hence, using equivalence between parts (i) and (iv) of Lemma 1, we see that (24) is nonoscillatory which contradicts our assumptions.

Remark 6. The oscillation criterion from Theorem 5 is general in the sense that the oscillation is given in terms of oscillation of a certain half-linear differential equation rather than in terms of explicit conditions on the coefficients of the equation. Most of the related papers continue the proofs by utilizing techniques used in the theory of half-linear ordinary differential equations (often simply copy of the proofs of known oscillation citeria) to reach effective conditions for oscillation. However, we feel our approach as an advantage, since it allows to utilize arbitrary from large family of oscillation criteria for half-linear oscillation equations to detect oscillation of delay equation. See also [8] for a comprehensive survey on oscillation criteria known up to 2005.

Remark 7. Note that a similar result like Theorem 5 can be proved also without Lemma 4 and using Lemma 2 instead. This results in a comparison of (1) with the equation

$$
\left(r(t) \Phi\left(x^{\prime}(t)\right)\right)^{\prime}+c(t) \lambda\left(\frac{\tau(t)}{t}\right)^{p-1} \Phi(x(t))=0,
$$

where $\lambda$ is a real parameter which satisfies $\lambda \in(0,1)$. (Note that for $r \equiv 1$ we get Theorem A.) Equation (24) can be viewed 
in a certain sense as a continuation of (26) with respect to $\lambda$ to the border value $\lambda=1$. Note that the problems related to oscillation of equation of the type (26) and dependence of oscillatory properties on the parameter $\lambda$ are referred to as conditional oscillation. In general, oscillation of (26) implies oscillation of (24), but the opposite implication need not be true in general, see the paper [17] which (based on the results from [18]) suggests a method on how to construct a pair of equations of the type (24) and (26) with (24) oscillatory and (26) nonoscillatory.

Remark 8. Theorem 5 extends Theorem A, where oscillation of (1) is deduced from oscillation of (26). The following example shows that this extension is nonempty.

Example 9. Consider the perturbed Euler type half-linear delay differential equation

$$
\begin{aligned}
\left(\Phi\left(x^{\prime}\right)\right)^{\prime} & +\left(\frac{p-1}{p}\right)^{p}\left(\frac{1}{t^{p}}+\frac{\mu}{t^{p} \ln t}\right) \\
& \times\left(\frac{t}{\tau(t)}\right)^{p-1} \Phi(x(\tau(t)))=0,
\end{aligned}
$$

where $\mu>0$ is real constant. According to Theorem 5, (27) is oscillatory if

$$
\left(\Phi\left(x^{\prime}\right)\right)^{\prime}+\left(\frac{p-1}{p}\right)^{p}\left(\frac{1}{t^{p}}+\frac{\mu}{t^{p} \ln t}\right) \Phi(x(t))=0
$$

is oscillatory. Following [8, Theorem 5.2.2] (see also [19]) we treat (28) as a perturbation of the nonoscillatory equation

$$
\left(\Phi\left(x^{\prime}\right)\right)^{\prime}+\left(\frac{p-1}{p}\right)^{p} \frac{1}{t^{p}} \Phi(x)=0
$$

with principal solution $h(t)=t^{(p-1) / p}$. A simple computation shows

$$
\int^{\infty}\left(\frac{p-1}{p}\right)^{p} \frac{\mu}{t^{p} \ln t} t^{p-1} d t=\infty
$$

hence (28) is oscillatory by [ 8 , Theorem 5.2.2]. Consequently, (27) is oscillatory for every $\mu$.

We claim that the oscillation of (27) cannot be proved with Theorem A. Really, in our example (11) becomes

$$
\left(\Phi\left(x^{\prime}\right)\right)^{\prime}+\lambda\left(\frac{p-1}{p}\right)^{p}\left(\frac{1}{t^{p}}+\frac{\mu}{t^{p} \ln t}\right) \Phi(x(t))=0,
$$

where $\lambda \in(0,1)$. This equation is nonoscillatory for every $\mu>0$ by Kneser type nonoscillation criterion [8, Theorem 1.4.5], and thus Theorem A fails to apply.

\section{Oscillation of Neutral Differential Equation}

In this section we use a slight modification of the estimates from the first part of the paper to derive similar results for the second order neutral differential equation

$$
\left(r(t) \Phi\left(z^{\prime}(t)\right)\right)^{\prime}+c(t) \Phi(x(\tau(t)))=0
$$

where

$$
z(t)=x(t)+a(t) x(\theta(t))
$$

$0 \leq a(t)<1, r(t)>0, c(t) \geq 0, \tau(t) \leq t, \theta(t) \leq t$, $\lim _{t \rightarrow \infty} \tau(t)=\lim _{t \rightarrow \infty} \theta(t)=\infty$.

Similarly as for (1), if $x$ is a solution of (32) on $\left[t_{0}, \infty\right)$ such that $z(t)$ is positive on $\left[t_{0}, \infty\right)$, then the function $w(t)=$ $r(t)\left(\Phi\left(z^{\prime}(t)\right) / \Phi(z(t))\right)$ satisfies the Riccati type equation

$$
w^{\prime}+c(t) \Phi\left(\frac{x(\tau(t))}{z(t)}\right)+(p-1) r^{1-q}(t)|w|^{q}=0
$$

on $\left[t_{0}, \infty\right)$.

Similarly like for the delay equation, the positive solution is increasing and concave down. More precisely, the following lemma holds. For linear version of this lemma see [2, Lemma 1] and for $p \geq 2$ see [1, Lemma 2.1].

Lemma 10. Let $x(t)$ be an eventually nonoscillatory solution of (32). If $\int^{\infty} r^{1-q}(t) d t=\infty$, then the corresponding function $z(t)=x(t)+a(t) x(\theta(t))$ satisfies

$$
z(t)>0, \quad z^{\prime}(t)>0
$$

eventually. Moreover, if $r^{\prime}(t) \geq 0$, then $z^{\prime \prime}(t)<0$ for large $t$.

Proof. The proof is essentially the same as the proof of $[1$, Lemma 2.1]. We just relax the restriction on $p$.

Without loss of generality we can suppose that $x$ is eventually positive solution of (32). There exists $T \in \mathbb{R}$ such that $x(t), x(\tau(t))$ and $x(\theta(t))$ are positive on $(T, \infty)$ and

$$
\left(r(t) \Phi\left(z^{\prime}(t)\right)\right)^{\prime}=-c(t) \Phi(x(\tau(t)))<0
$$

for $t \in(T, \infty)$. Hence, $r(t) \Phi\left(z^{\prime}(t)\right)$ is decreasing and either

$$
\Phi\left(z^{\prime}(t)\right)>0 \quad \text { or } \quad \Phi\left(z^{\prime}(t)\right)<0
$$

for large $t$.

Suppose that there exists $T_{1}>T$ such that $\Phi\left(z^{\prime}(t)\right)<0$ for $t \geq T_{1}$. There exists a positive constant $M$ such that

$$
\begin{aligned}
& r(t) \Phi\left(z^{\prime}(t)\right)<-M<0, \\
& z^{\prime}(t)<-\Phi^{-1}(M) r^{1-q}(t)
\end{aligned}
$$

for $t \geq T_{1}$. Integrating this inequality over the interval $\left(T_{1}, t\right)$ we get

$$
z(t) \leq z\left(T_{1}\right)-\Phi^{-1}(M) \int_{T_{1}}^{t} r^{1-q}(s) d s .
$$

Letting $t \rightarrow \infty$ we have a negative upper bound for the function $z$ and large $t$. However, the positivity of both $x(t)$ and $x(\theta(t))$ implies positivity of $z$. This contradiction proves that $\Phi\left(z^{\prime}(t)\right)>0$ and $z^{\prime}(t)>0$ eventually.

$$
\begin{aligned}
& \text { If } r^{\prime}(t) \geq 0 \text {, then } \\
& \begin{aligned}
0> & \left(r(t) \Phi\left(z^{\prime}(t)\right)\right)^{\prime} \\
& =r^{\prime}(t) \Phi\left(z^{\prime}(t)\right)+r(t)(p-1)\left|z^{\prime}(t)\right|^{p-2} z^{\prime \prime}(t) \\
& \geq r(t)(p-1)\left|z^{\prime}(t)\right|^{p-2} z^{\prime \prime}(t),
\end{aligned}
\end{aligned}
$$

and hence $z^{\prime \prime}(t)<0$. 
Lemma 11. Suppose that $x$ is an eventually positive nonoscillatory solution of (32) and $z$ is the corresponding function defined by (33). If $\int^{\infty} r^{1-q}(t) d t=\infty$, then

$$
x(\tau(t)) \geq[1-a(\tau(t))] z(\tau(t))
$$

eventually.

Proof. According to Lemma 10 there exists $T$ such that

$$
x(\theta(\theta(t)))>0, \quad z(t)>0, \quad z^{\prime}(t)>0
$$

holds for $t \geq \tau(T)$. From here and from the fact that $z$ is increasing and $\theta$ is delay we have

$$
\begin{aligned}
z(t) & =x(t)+a(t) x(\theta(t)) \leq x(t)+a(t) z(\theta(t)) \\
& \leq x(t)+a(t) z(t) .
\end{aligned}
$$

From here we conclude

$$
z(t)(1-a(t)) \leq x(t)
$$

and hence (41) holds for $t>T$.

The following lemma is an alternative to Lemma 4 for neutral differential equations.

Lemma 12. Suppose that (32) is nonoscillatory and $x(t)$ is an eventually positive solution of (32). If

$$
\int^{\infty} c(s)(1-a(\tau(s)))^{p-1}(\tau(s))^{p-1} d s=\infty
$$

and (14) holds, then the function $z(t) / t$ is decreasing eventually.

Proof. Similarly like in Lemma 4 we find the derivative

$$
\left(\frac{z(t)}{t}\right)^{\prime}=\frac{z^{\prime}(t) t-z(t)}{t^{2}}
$$

It is sufficient to show that $z^{\prime}(t) t-z(t)<0$ eventually. Lemma 10 implies that there exists $t_{0}$ such that $z^{\prime \prime}(t)<0$ on $\left(t_{0}, \infty\right)$. This shows that $z^{\prime}(t) t-z(t)$ is decreasing on $\left(t_{0}, \infty\right)$. As a consequence, if $z^{\prime}\left(t_{1}\right) t_{1}-z\left(t_{1}\right)<0$ for some $t_{1}>t_{0}$, then $z^{\prime}(t) t-z(t)<0$ on $\left(t_{1}, \infty\right)$.

Suppose by contradiction that there exists $t_{2}$ such that $z^{\prime}(t) t-z(t)>0$ on $\left(t_{2}, \infty\right)$. Solving this inequality we get

$$
z(t) \geq \frac{z\left(t_{2}\right)}{t_{2}} t
$$

Now integrating (32) from $t_{2}$ to $t$ and using (41) we get

$$
\begin{aligned}
r(t) \Phi\left(z^{\prime}(t)\right) & =r\left(t_{2}\right) \Phi\left(z^{\prime}\left(t_{2}\right)\right)-\int_{t_{2}}^{t} c(s)(x(\tau(s)))^{p-1} d s \\
& \leq r\left(t_{2}\right) \Phi\left(z^{\prime}\left(t_{2}\right)\right)
\end{aligned}
$$

$$
\begin{aligned}
& -\int_{t_{2}}^{t} c(s)[1-a(\tau(s))]^{p-1}(z(\tau(s)))^{p-1} d s \\
\leq & r\left(t_{2}\right) \Phi\left(z^{\prime}\left(t_{2}\right)\right)-\frac{z\left(\tau\left(t_{2}\right)\right)}{\tau\left(t_{2}\right)} \\
& \times \int_{t_{2}}^{t} c(s)[1-a(\tau(s))]^{p-1}(\tau(s))^{p-1} d s .
\end{aligned}
$$

Taking $t$ sufficiently large and using (45) we obtain a negative upper bound for a positive function $r(t) \Phi\left(z^{\prime}(t)\right)$. This contradiction proves the lemma.

Now we can formulate the comparison theorem which relates neutral differential equations to ordinary secondorder half-linear differential equations.

Theorem 13. Suppose that (45) and (14) hold. If the ordinary half-linear differential equation

$$
\begin{aligned}
& \left(r(t) \Phi\left(x^{\prime}(t)\right)\right)^{\prime}+c(t)[1-a(\tau(t))]^{p-1} \\
& \times\left(\frac{\tau(t)}{t}\right)^{p-1} \Phi(x(t))=0
\end{aligned}
$$

is oscillatory, then (32) is also oscillatory.

Proof. Having proved important estimates in the preceding two lemmas, the proof of the theorem is a modification of the proof of Theorem 5 . If $x(t)$ is an eventually positive solution of (32), then the function $w$ defined by $w(t)=$ $r(t) \Phi\left(z^{\prime}(t)\right) / \Phi(z(t))$ satisfies (34). Using Lemmas 11 and 12 we see that

$$
\begin{aligned}
0= & w^{\prime}+c(t) \Phi\left(\frac{x(\tau(t))}{z(t)}\right)+(p-1) r^{1-q}(t)|w|^{q} \\
\geq & w^{\prime}+c(t)[1-a(\tau(t))]^{p-1} \Phi\left(\frac{z(\tau(t))}{z(t)}\right) \\
& +(p-1) r^{1-q}(t)|w|^{q} \\
\geq & w^{\prime}+c(t)[1-a(\tau(t))]^{p-1}\left(\frac{\tau(t)}{t}\right)^{p-1} \\
& +(p-1) r^{1-q}(t)|w|^{q} .
\end{aligned}
$$

Hence (49) is nonoscillatory by Lemma 1.

Remark 14. A version of Theorem 13 has been used implicitly in the proof of [20, Theorem 2.2] for dynamic equations. A closer estimation of the proof shows that one of the important steps is an application of inequality which in the continuous case reads as (10). However, Lemma 12 allows the estimate

$$
\frac{z(\tau(t))}{z(t)} \geq \frac{\tau(t)}{t}
$$

which appears to be sharper, since

$$
\frac{1-(T / \tau(t))}{1-(T / t)} \leq 1
$$


and the annoying dependence of the left-hand side on $T$ usually necessitates to replace it by a constant $k<1$ which may appear in the resulting oscillation criterion.

\section{Acknowledgment}

This research is supported by the Grant P201/10/1032 of the Czech Science Foundation.

\section{References}

[1] B. Baculíková, T. Li, and J. Džurina, "Oscillation theorems for second-order superlinear neutral differential equations," Mathematica Slovaca, vol. 63, no. 1, pp. 123-134, 2013.

[2] B. Baculíková and J. Džurina, "Oscillation theorems for second order neutral differential equations," Computers \& Mathematics with Applications, vol. 61, no. 1, pp. 94-99, 2011.

[3] B. Baculíková and J. Džurina, "Oscillation theorems for secondorder nonlinear neutral differential equations," Computers \& Mathematics with Applications, vol. 62, no. 12, pp. 4472-4478, 2011.

[4] R. P. Agarwal, D. R. Anderson, and A. Zafer, "Interval oscillation criteria for second-order forced delay dynamic equations with mixed nonlinearities," Computers \& Mathematics with Applications, vol. 59, no. 2, pp. 977-993, 2010.

[5] D. R. Anderson and A. Zafer, "Nonlinear oscillation of secondorder dynamic equations on time scales," Applied Mathematics Letters, vol. 22, no. 10, pp. 1591-1597, 2009.

[6] A. Tiryaki, Y. Başci, and I. Güleç, "Interval criteria for oscillation of second-order functional differential equations," Computers \& Mathematics with Applications, vol. 50, no. 8-9, pp. 1487-1498, 2005.

[7] A. Zafer, "Interval oscillation criteria for second order superhalf linear functional differential equations with delay and advanced arguments," Mathematische Nachrichten, vol. 282, no. 9, pp. 1334-1341, 2009.

[8] O. Došlý and P. Řehák, Half-Linear Differential Equations, vol. 202 of North-Holland Mathematics Studies, Elsevier, San Diego, Calif, USA, 2005.

[9] J. Ohriska, "Oscillation of second order delay and ordinary differential equation," Czechoslovak Mathematical Journal, vol. 34, no. 1, pp. 107-112, 1984.

[10] G. H. Hardy, J. E. Littlewood, and G. Polya, Inequalities, Cambridge University Press, Cambridge, UK, 1999.

[11] R. P. Agarwal, S.-L. Shieh, and C.-C. Yeh, "Oscillation criteria for second-order retarded differential equations," Mathematical and Computer Modelling, vol. 26, no. 4, pp. 1-11, 1997.

[12] R. Xu and F. Meng, "New Kamenev-type oscillation criteria for second order neutral nonlinear differential equations," Applied Mathematics and Computation, vol. 188, no. 2, pp. 1364-1370, 2007.

[13] Z. Opluštil and J. Šremr, "Some oscillation criteria for the second-order linear delay differential equation," Mathematica Bohemica, vol. 136, no. 2, pp. 195-204, 2011.

[14] Z. Opluštil and J. Šremr, "On oscillations of solutions to secondorder linear delay differential equations," Georgian Mathematical Journal, vol. 20, no. 1, pp. 65-94, 2013.

[15] L. Erbe, A. Peterson, and S. H. Saker, "Kamenev-type oscillation criteria for second-order linear delay dynamic equations," Dynamic Systems and Applications, vol. 15, no. 1, pp. 65-78, 2006.
[16] J. Džurina and I. P. Stavroulakis, "Oscillation criteria for secondorder delay differential equations," Applied Mathematics and Computation, vol. 140, no. 2-3, pp. 445-453, 2003.

[17] P. Hasil and M. Veselý, "Oscillation of half-linear differential equations with asymptotically almost periodic coefficients," Advances in Difference Equations, vol. 2013, article 122, 15 pages, 2013.

[18] M. Veselý, "Construction of almost periodic functions with given properties," Electronic Journal of Differential Equations, no. 29, pp. 1-25, 2011.

[19] Á. Elbert, "Oscillation and nonoscillation theorems for some non-linear ordinary differential equations," in Ordinary and Partial Differential Equations, vol. 964 of Lecture Notes in Mathematics, pp. 187-212, 1982.

[20] S. H. Saker and D. O’Regan, "New oscillation criteria for second-order neutral functional dynamic equations via the generalized Riccati substitution," Communications in Nonlinear Science and Numerical Simulation, vol. 16, no. 1, pp. 423-434, 2011. 


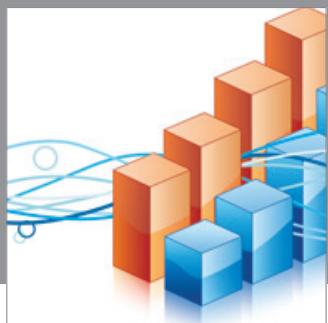

Advances in

Operations Research

mansans

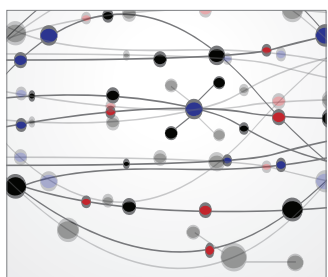

The Scientific World Journal
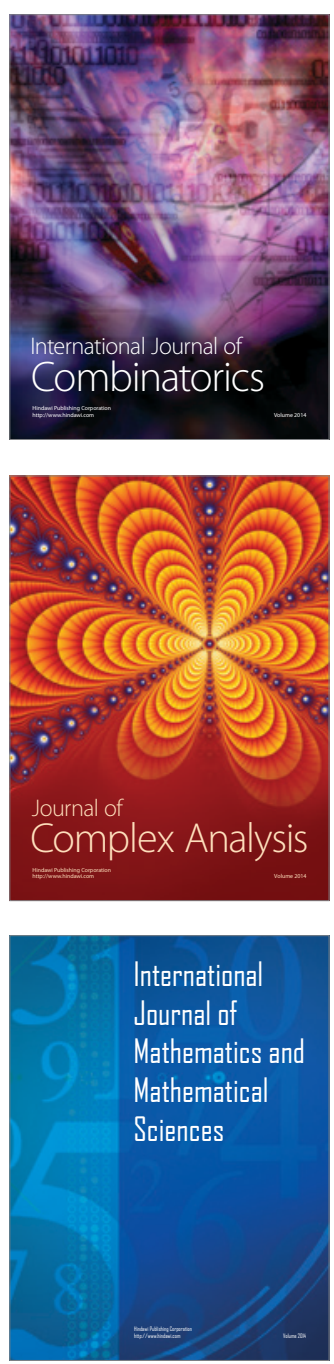
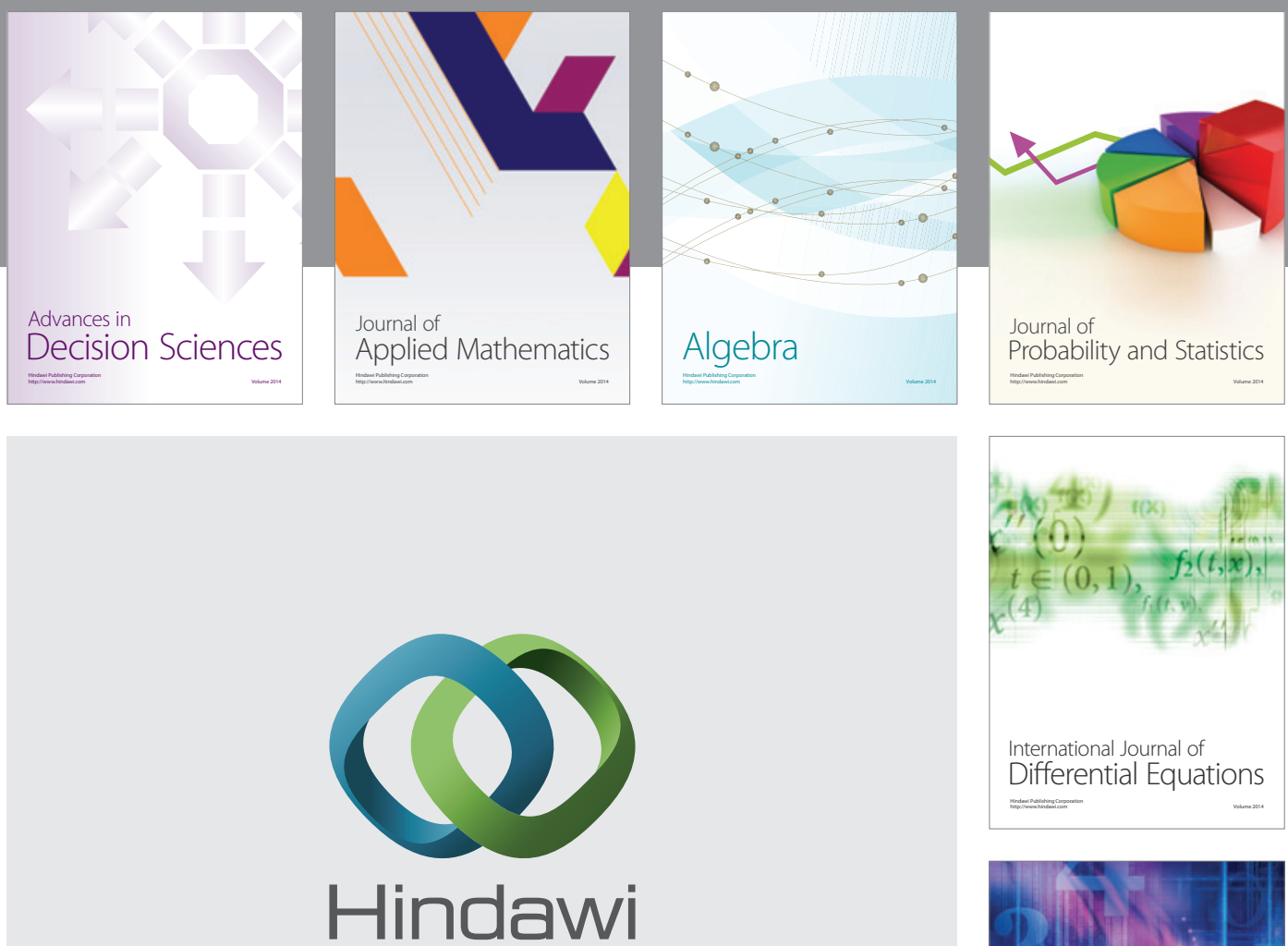

Submit your manuscripts at http://www.hindawi.com
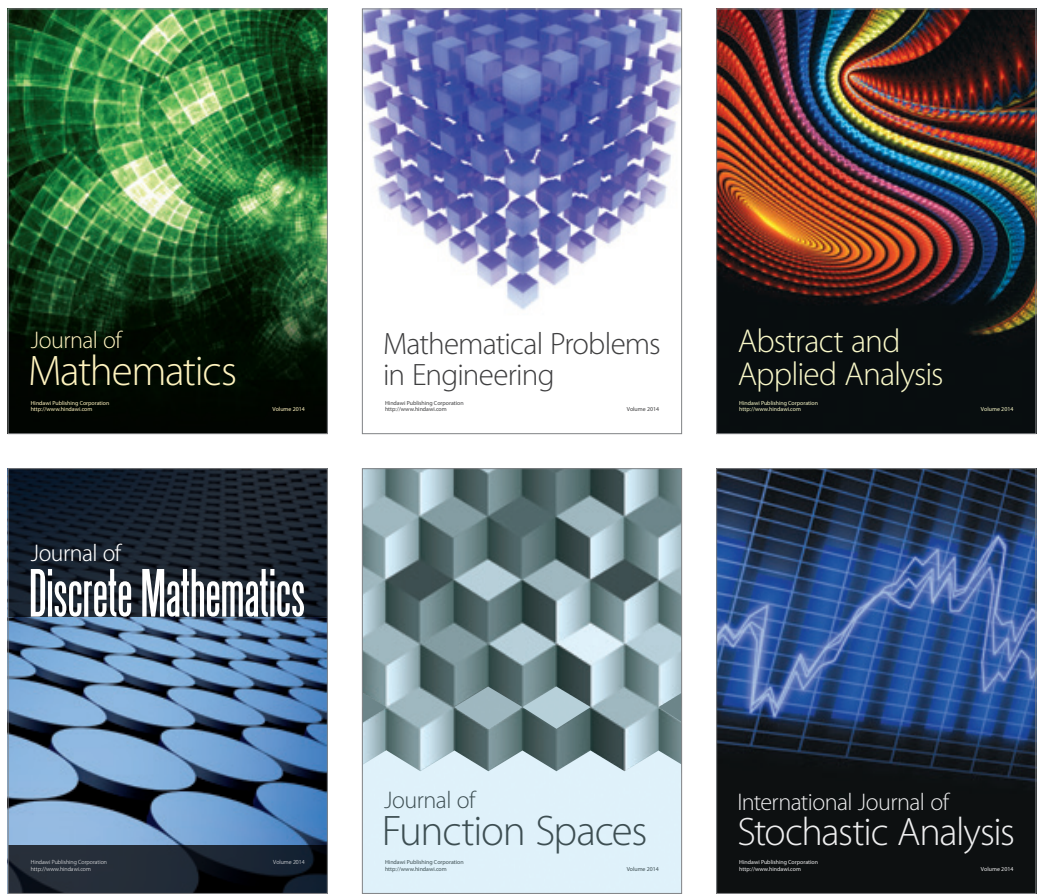

Journal of

Function Spaces

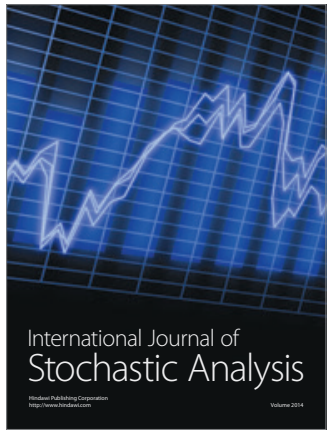

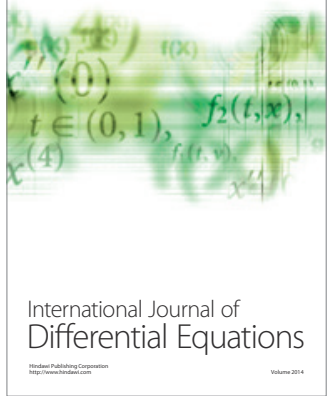
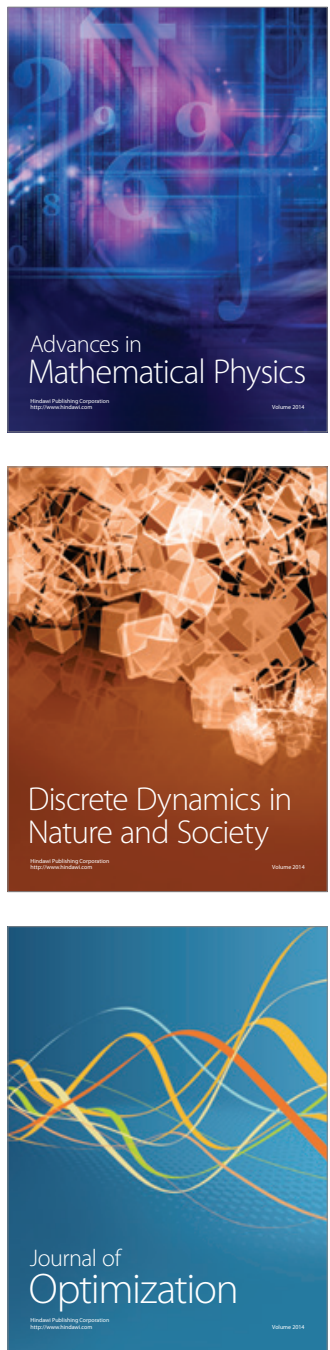